\title{
Mutation screening analysis of the retinoblastoma related gene RB2/p130 in sporadic ovarian cancer and head and neck squamous cell cancer
}

\author{
A J Alvi, R Hogg, J S Rader, M J Kuo, E R Maher, F Latif
}

J Clin Pathol: Mol Pathol 2002;55:153-155

See end of article for authors' affiliations

.......................

Correspondence to: Dr F Latif, Section of Medical and Molecular

Genetics, Department of

Paediatrics and Child

Health, University of

Birmingham, Birmingham

B15 2TT, UK;

flatif@hgmp.mrc.ac.uk

Accepted for publication 20 November 2001

\begin{abstract}
Aims: To investigate the involvement of the RB2/p130 gene in the pathogenesis of sporadic ovarian cancer in addition to head and neck squamous cell carcinoma (HNSCC).

Methods: Paired tumour and patient matched normal DNA samples from 43 sporadic ovarian tumours and 39 normal/tumour HNSCC DNA samples were screened. The mutation screen used polymerase chain reaction (PCR) amplification followed by single strand conformation polymorphism analysis and direct sequencing of the PCR products. Exons 19 and 20 (B domain) and exons 21 and 22 (C-terminus) were analysed for mutations. These exons were chosen because most of the point mutations in RB2/ p130 are located in the C-terminal region and mutations in these exons have been identified previously in nasopharyngeal carcinomas and primary lung tumours.

Results: No abnormal band shifts were seen in the samples analysed, and no bands directly sequenced revealed the presence of mutations.

Conclusions: Genetic alterations in the RB2/p130 gene (exons 19-22) are unlikely to be involved directly in the pathogenesis of sporadic ovarian cancer or HNSCC.
\end{abstract}

Abbreviations: HNSCC, head and neck squamous cell carcinoma; $\mathrm{LOH}$, loss of heterozygosity; NLS, nuclear localisation signal; PCR, polymerase chain reaction; $\mathrm{pRb}$, retinoblastoma protein; $\mathrm{Rb}$, retinoblastoma gene family; SSCP, single strand conformational polymorphism 


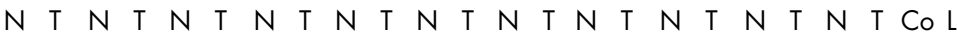

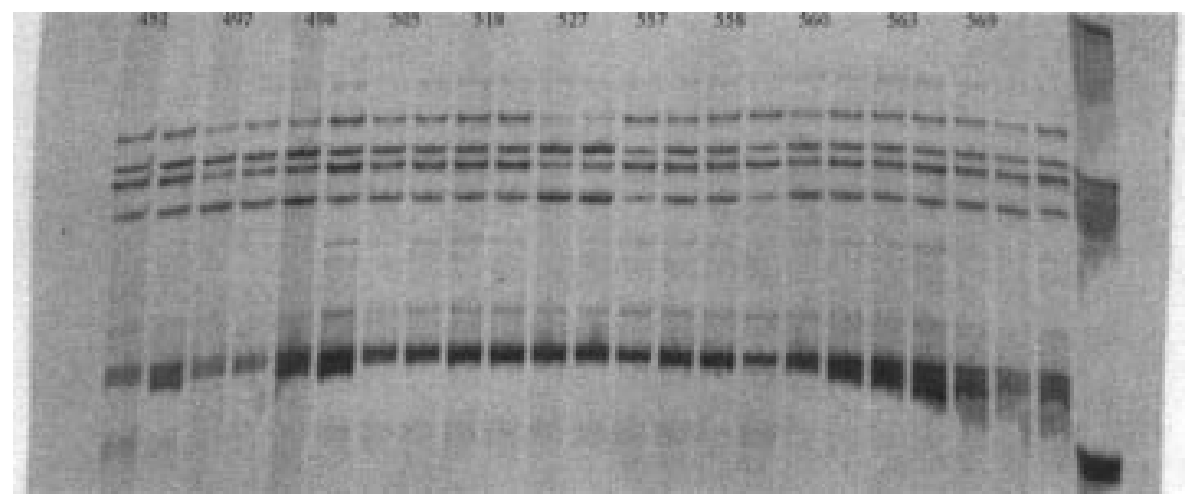

Figure 1 Single strand conformational polymorphism (SSCP) analysis of RB2/p120 exon 22 in matched primary ovarian tumour/normal pairs (the case number for each pair is shown at the top). N, normal; T, tumour; Co, control genomic DNA sample; L, DNA ladder. gene. ${ }^{78}$ They also reported mutations leading to abnormal protein localisation in lymphoid cell lines through disruption of the NLS, ${ }^{9}$ in addition to providing strong evidence to support the role of RB2/p130 as a tumour suppressor gene in lung cancer. ${ }^{8}$

\section{"All three retinoblastoma gene family members display growth suppressive activities, which result in the blocking of cells in the Gl phase of the cell cycle"}

Cytogenetic studies have permitted the definition of chromosomal areas commonly displaying loss of heterozygosity (LOH) in tumour cells. A previous study of ovarian cancer suggested the involvement of one or more tumour suppressor genes on several chromosomes, ${ }^{10}$ in particular, chromosome 16q, the location of the putative tumour suppressor RB2/p130. The role of this gene in the pathogenesis of ovarian cancer was suggested by frequent $\mathrm{LOH}(38-67 \%)$ at this chromosomal region. ${ }^{10}{ }^{11}$ Recently, it was reported that $17 \%$ of HNSCCs also showed LOH at chromosome 16q. ${ }^{12}$ In addition, Claudio and colleagues ${ }^{7}$ found mutations in $\mathrm{RB} 2 / \mathrm{pl} 30$ in a significant proportion of nasopharyngeal carcinomas, a subset of HNSCC.

To investigate a possible role for the RB2/p130 gene in the pathogenesis of sporadic ovarian and head and neck cancers we undertook a mutation screen analysis of the RB2/p130 gene from exons 19 to 22 in these cancers.

\section{MATERIALS AND METHODS \\ DNA samples}

Ovarian tumours and patient matched normal DNA sample pairs (43 pairs) were obtained from the Washington University School of Medicine (St Louis). Patient samples represented a wide range of tumour types and stages (stages I to IV epithelial tumours including 13 serous, eight endometrioid, eight mixed histological, four teratomas, three mucinous, two granulosas, two unknown, one clear cell, one fibroadenoma, and one unclassified sex cord). None of the family histories suggested a hereditary component in these patients. Normal/ tumour pairs from patients with HNSCC (39 pairs: 18 laryngeal, 12 pharyngeal, seven oral cavity, and two paranasal sinuses), which included a full range of tumour stages and had been analysed for known clinicopathological parameters, were collected at the Queen Elizabeth Medical Hospital (Birmingham, UK).

\section{PCR and mutation analysis}

Tumour and normal DNA pairs were amplified by the polymerase chain reaction (PCR) using previously published primers. $^{7}$ Exons 19, 20, and 22 of RB2/p130 were amplified using identical PCR programmes, which comprised one cycle of five minutes at $95^{\circ} \mathrm{C}$ and 38 cycles of 30 seconds at each of $95^{\circ} \mathrm{C}, 55^{\circ} \mathrm{C}$, and $72^{\circ} \mathrm{C}$, followed by one cycle of seven minutes at $72^{\circ} \mathrm{C}$. Standard Taq polymerase was used for these PCRs (Gibco/Life Technologies, Paisley, UK). For PCR analysis of exon 21, a proofreading Taq polymerase (DyNAzyme; Flowgen) was used as follows: one cycle of denaturation at $95^{\circ} \mathrm{C}$ for five minutes, 35 cycles consisting of one minute at $95^{\circ} \mathrm{C}$, one minute at $55^{\circ} \mathrm{C}$, and one minute at $72^{\circ} \mathrm{C}$, followed by one cycle at $72^{\circ} \mathrm{C}$ for five minutes (for exon 21 multiple bands were obtained with standard Taq polymerase so proofreading Taq polymerase was used).

For mutation analysis, $2 \mu \mathrm{l}$ of each PCR product was mixed with $2 \mu \mathrm{l}$ of loading buffer and denatured for 10 minutes at $96^{\circ} \mathrm{C}$. The samples were then run in a cold chamber on $8 \%$ non-denaturing single strand conformational polymorphism (SSCP) polyacrylamide gels (Accugel 19/1; National Diagnostics, Hessle, Yorkshire, UK) containing 5\% glycerol for 16-18 hours at 160-180 V, followed by silver staining and drying of the gels on to Whatman paper. Direct sequencing was performed on an ABI 377 automated sequencer using a dRhodamine cycle sequencing kit (PE Applied Biosystems, Warrington, UK).

\section{RESULTS}

\section{Analysis of the RB2/p130 gene in ovarian cancer and HNSCC}

To investigate whether mutations in the RB2/pl30 gene could play a role in sporadic ovarian cancer and HNSCC, we analysed a total of 82 tumour/normal DNA pairs for mutations in exons 19-22 of this gene. Exons 19-22 span the region that encodes the $\mathrm{B}$ domain and C-terminus of the protein where the putative NLS is located. These exons were chosen on the basis of previous work, which identified mutations resulting in either frameshifts following insertions or point substitutions in nasopharyngeal carcinoma, ${ }^{7}$ or abnormal localisation of the $\mathrm{Rb}$ protein following disruption of the NLS in lymphoid cell lines.' All four exons were amplified by PCR and were first analysed on an SSCP gel (fig 1). No abnormalities were seen upon gel analysis. Positive controls were not available for the SSCP analysis; therefore, we then directly sequenced the samples to ensure that we had not missed any shifts on SSCP as a result of false negativity. No changes were found upon direct sequencing of the exons (all ovarian samples were sequenced for exons 19-22, a third of head and neck samples were also directly sequenced). Thus, no mutations were found in the exons analysed for the 43 ovarian and 39 HNSCC tumour samples.

\section{DISCUSSION}

The importance of the Rb pathway of cell proliferation regulation is underlined by the finding that most sporadic human cancers carry mutations either in Rb or in one of the other key components of the pathway. RBl is the prototype tumour suppressor gene. In humans, retinoblastomas can arise after 


\section{Take home messages}

- No abnormal band shifts were seen in the samples of ovarian and head and neck squamous cell carcinoma analysed and no mutations in the RB2/p130 gene (exons 19-22) by direct sequencing

- Thus, genetic alterations in the RB2/p130 gene lexons 19-22) are unlikely to be involved directly in the pathogenesis of sporadic ovarian cancer or head and neck squamous cell carcinoma

the loss of RBl function through inactivating mutations and $\mathrm{LOH}$. In the case of inherited retinoblastoma susceptibility, the defective allele is inherited through the germline. RBI and the two other members of the Rb family, pl07 and RB2/p130, perform overlapping but not fully redundant functions in cell cycle regulation and cell growth inhibition. The involvement of p107 mutations has not yet been reported for human cancers, but in the case of RB2/p130 pathological mutations are beginning to be identified. Recently, mutations disrupting the normal nuclear localisation of the $\mathrm{pRb} 2 / \mathrm{pl} 30$ protein in lymphoid cell lines were identified, ${ }^{9}$ in addition to mutations affecting the normal functioning of RB2/p130 in nasopharyngeal tumours. ${ }^{7}$ Furthermore, the role of RB2/p130 as a tumour suppressor gene is strongly supported by the finding of tumour regression in nude mice treated with wild-type RB2/p130 after the development of lung tumours. ${ }^{8}$

This putative tumour suppressor gene is located on the long arm of chromosome 16, an area displaying a high degree of $\mathrm{LOH}$ in several tumour types, notably ovarian and breast cancers, but also HNSCC. LOH of chromosome 16q has been reported in $38-67 \%$ of ovarian tumours ${ }^{10}{ }^{11}$ and $17 \%$ of HNSCCs. ${ }^{12}$ In the same report by Sato et al,,$^{10} 39 \%$ of ovarian tumours also showed $\mathrm{LOH}$ for $17 \mathrm{q}$, where subsequently the breast/ovarian tumour susceptibility gene, BRCAl, was identified. However, other reports showing a high degree of $\mathrm{LOH}$ of chromosome 13 (where RBl resides) in high grade ovarian carcinomas also found normal nuclear $\mathrm{Rb}$ protein expression, suggesting that RBI was not the target of allelic loss and that other $13 \mathrm{q}$ genes were involved in malignant transformation. ${ }^{13}{ }^{14}$ In our present study, none of our 43 ovarian tumour samples had mutations in crucial areas of the RB2/p130 gene. The same was true for our series of 39 HNSCCs (our set of HNSCCs included only one nasopharangeal carcinoma), indicating that mutations in exons 19-22 of the RB2/p130 gene may not play a role in the aetiology of nonnasopharangeal carcinoma head and neck carcinomas and ovarian tumours.

"Loss of heterozygosity of chromosome $16 \mathrm{q}$ has been reported in $38-67 \%$ of ovarian tumours and $17 \%$ of head and neck squamous cell carcinomas"
It must be mentioned that the SSCP technique does not identify all of the mutations that may be present. However, many of our samples were directly sequenced and still no mutations were found. We conclude that mutations in exons 19-22 of the $\mathrm{RB} 2 / \mathrm{pl} 30$ gene are not an important factor in the pathogenesis of these cancer types and that other as yet unidentified genes located on chromosome 16q may play a more important role.

\section{ACKNOWLEDGEMENTS}

This work was supported in part by Wellbeing and Get A-Head charity. RPH is a recipient of a fellowship from Royal College of Surgeons of England (Newman Fellowship).

\section{Authors' affiliations}

A J Alvi, R Hogg, E R Maher, F Latif, Section of Medical and Molecular Genetics, Department of Paediatrics and Child Health, University of Birmingham, Birmingham B15 2TT, UK

M J Kuo, Department of Otolaryngology and Head and Neck Surgery,

Queen Elizabeth Hospital, Birmingham, UK

J S Rader, Department of Obstetrics and Gynecology, Washington

University School of Medicine, St Louis, Missouri 63110, USA

\section{REFERENCES}

1 Berchuck A, Schildkraut JM, Marks JR, et al. Managing hereditary ovarian cancer risk. Cancer 1999:86:1697-704.

2 Rubin SC, Blackwood MA, Bandera C, et al. BRCA1, BRCA2, and hereditary nonpolyposis colorectal cancer gene mutations in an unselected ovarian cancer population: relationship to family history and implications for genetic testing. Am J Obstet Gynecol 1998;178:670-7.

3 Vokes EE, Weichselbaum RR, Lippman SM, et al. Head and neck cancer. N Engl J Med 1993;328:184-94.

4 Saranath D, Bhoite LT, Deo MG. Molecular lesions in human oral cancer: the Indian scene. Eur J Cancer B Oral Oncol 1993;29B: 107-12. 5 Weinberg RA. The retinoblastoma protein and cell cycle control. Cell 1995;81:323-30.

6 Baldi A, Boccia V, Claudio PP, et al. Genomic structure of the human retinoblastoma-related Rb2/p130 gene. Proc Natl Acad Sci U S A 1996;93:4629-32

7 Claudio PP, Howard CM, Fu Y, et al. Mutations in the retinoblastoma-related gene $\mathrm{RB} 2 / \mathrm{p} 130$ in primary nasopharyngeal carcinoma. Cancer Res 2000:60:8-12.

8 Claudio PP, Howard CM, Pacilio C, et al. Mutations in the retinoblastoma-related gene RB2/p130 in lung tumors and suppression of tumor growth in vivo by retrovirus-mediated gene transfer. Cancer Res $2000 ; 60: 372-82$

9 Cinti C, Claudio PP, Howard CM, et al. Genetic alterations disrupting the nuclear localization of the retinoblastoma-related gene RB2/p 130 in human tumor cell lines and primary tumors. Cancer Res 2000;60:383-9.

10 T Sato, H Saito, R Morita, et al. Allelotype of human ovarian cancer. Cancer Res 1991:51:5118-22.

11 Kawakami M, Staub J, Cliby W, et al. Involvement of H-cadherin $(\mathrm{CDH} 13)$ on $16 \mathrm{q}$ in the region of frequent deletion in ovarian cancer. Int J Oncol 1999;15:715-20.

12 Xang X, Gleich L, Pavelic ZP, et al. Cervical metastases of head and neck squamous cell carcinoma correlate with loss of heterozygosity on chromosome 16q. Int J Oncol 1999:14:557-61.

$13 \mathrm{Kim} \mathrm{TM}$, Benedict WF, Xu HJ, et al. Loss of heterozygosity on chromosome 13 is common only in the biologically more aggressive subtypes of ovarian epithelial tumors and is associated with normal retinoblastoma gene expression. Cancer Res 1994;54:605-9.

14 Liu Y, Heyman M, Wang Y, et al. Molecular analysis of the retinoblastoma gene in primary ovarian cancer cells. Int J Cancer 1994;58:663-7. 